\title{
On locally contractive fuzzy set-valued mappings
}

\author{
Jamshaid Ahmad ${ }^{1}$, Akbar Azam ${ }^{1}$ and Salvador Romaguera ${ }^{2 *}$
}

"Correspondence:

sromague@mat.upv.es

${ }^{2}$ Instituto Universitario de

Matemática Pura y Aplicada,

Universitat Politècnica de València, Valencia, 46022, Spain

Full list of author information is

available at the end of the article

\begin{abstract}
We prove the existence of common fuzzy fixed points for a sequence of locally contractive fuzzy mappings satisfying generalized Banach type contraction conditions in a complete metric space by using iterations. Our main result generalizes and unifies several well-known fixed-point theorems for multivalued maps. Illustrative examples are also given.
\end{abstract}

MSC: 46S40;47H10;54H 25

Keywords: fixed point; fuzzy mapping; contractive mapping; locally contractive

\section{Introduction}

The Banach contraction theorem and its subsequent generalizations play a fundamental role in the field of fixed point theory. In particular, Heilpern introduced in [1] the notion of a fuzzy mapping in a metric linear space and proved a Banach type contraction theorem in this framework. Subsequently several other authors [2-10] have studied and established the existence of fixed points of fuzzy mappings. The aim of this paper is to prove a common fixed-point theorem for a sequence of fuzzy mappings in the context of metric spaces without the assumption of linearity. Our results generalize and unify several typical theorems of the literature.

\section{Preliminaries}

Given a metric space $(X, d)$, denote by $C B(X)$ the family of all nonempty closed bounded subsets of $(X, d)$. As usual, for $\zeta \in X$ and $A \in C B(X)$, we define

$$
d(\zeta, A)=\inf _{a \in A} d(\zeta, a)
$$

Then the Hausdorff metric $H$ on $C B(X)$ induced by $d$ is defined as

$$
H(A, B)=\max \left\{\sup _{a \in A} d(a, B), \sup _{b \in B} d(A, b)\right\}
$$

for all $A, B \in C B(X)$.

A fuzzy set in $(X, d)$ is a function with domain $X$ and values in $I=[0,1] \cdot I^{X}$ denotes the collection of all fuzzy sets in $X$. If $A$ is a fuzzy set and $\zeta \in X$, then the function value $A(\zeta)$ is called the grade of membership of $\zeta$ in $A$. The $\alpha$-level set of a fuzzy set $A$ is denoted by

\section{Springer}

(o2014 Ahmad et al.: licensee Springer. This is an Open Access article distributed under the terms of the Creative Commons Attribution License (http://creativecommons.org/licenses/by/2.0), which permits unrestricted use, distribution, and reproduction in any medium, provided the original work is properly cited. 
$A_{\alpha}$, and it is defined as follows:

$$
\begin{aligned}
& A_{\alpha}=\{\zeta: A(\zeta) \geq \alpha\} \quad \text { if } \alpha \in(0,1], \\
& A_{0}=\text { closure of }\{\zeta: A(\zeta)>0\} .
\end{aligned}
$$

According to Heilpern [1], a fuzzy set $A$ in a metric linear space $(X, d)$ is said to be an approximate quantity if $A_{\alpha}$ is compact and convex in $X$, for each $\alpha \in(0,1]$, and $\sup _{\zeta \in X} A(\zeta)=1$. The family of all approximate quantities of the metric linear space $(X, d)$ is denoted by $W(X)$.

Now, for $A, B \in W(X)$ and $\alpha \in[0,1]$, define

$$
D_{\alpha}(A, B)=H\left(A_{\alpha}, B_{\alpha}\right)
$$

and

$$
d_{\infty}(A, B)=\sup _{\alpha \in[0,1]} D_{\alpha}\left(A_{\alpha}, B_{\alpha}\right)
$$

It is well known that $d_{\infty}$ is a metric on $W(X)$.

In case that $(X, d)$ is a (non-necessarily linear) metric space, we also define

$$
D_{\alpha}(A, B)=H\left(A_{\alpha}, B_{\alpha}\right),
$$

whenever $A, B \in I^{X}$ and $A_{\alpha}, B_{\alpha} \in C B(X), \alpha \in[0,1]$.

In the sequel the letter $\mathbb{N}$ will denote the set of positive integer numbers.

The following well-known properties on the Hausdorff metric (see e.g. [11]) will be useful in the next section.

Lemma 2.1 Let $(X, d)$ be a metric space and let $A, B \in C B(X)$ with $H(A, B)<r, r>0$. If $a \in A$, then there exists $b \in B$ such that $d(a, b)<r$.

Lemma 2.2 Let $(X, d)$ be a metric space and let $\left\{A_{n}\right\}_{n=1}^{\infty}$ be a sequence in $C B(X)$ such that $\lim _{n \rightarrow \infty} H\left(A_{n}, A\right)=0$, for some $A \in C B(X)$. If $\xi_{n} \in A_{n}$, for all $n \in \mathbb{N}$, and $d\left(\xi_{n}, \xi\right) \rightarrow 0$, then $\xi \in A$.

Now, let $X$ be an arbitrary set and let $Y$ be a metric space. A mapping $T$ is called fuzzy mapping if $T$ is a mapping from $X$ into $I^{Y}$. In fact, a fuzzy mapping $T$ is a fuzzy subset on $X \times Y$ with membership function $T(\zeta)$. The value $T(\zeta)(\xi)$ is the grade of membership of $\xi$ in $T(\zeta)$.

If $(X, d)$ is a metric space and $T$ is a (fuzzy) mapping from $X$ into $I^{X}$, we say that $\xi \in X$ is a fixed point of $T$ if $\xi \in T(\xi)_{1}$.

We conclude this section with the notion of contractiveness that will be used in our main result.

Definition 2.3 (compare [12]) Let $\varepsilon \in(0, \infty]$. A function $\psi:[0, \varepsilon) \rightarrow[0,1)$ is said to be a $M T$-function if it satisfies Mizoguchi-Takahashi's condition (i.e., $\limsup _{r \rightarrow t^{+}} \psi(r)<1$, for all $t \in[0, \varepsilon)$ ). 
Clearly, if $\psi:[0, \varepsilon) \rightarrow[0,1)$ is a nondecreasing function or a nonincreasing function, then it is a $M T$-function. So the set of $M T$-functions is a rich class.

\section{Fixed points of fuzzy mappings}

Fixed-point theorems for locally contractive mappings were studied, among others, by Edelstein [13], Beg and Azam [14], Holmes [15], Hu [11], Hu and Rosen [16], Ko and Tasi [17], Kuhfitting [18] and Nadler [19].

Heilpern [1] established a fixed-point theorem for fuzzy contraction mappings in metric linear spaces, which is a fuzzy extension of Banach's contraction principle. Afterwards Azam et al. [4, 5], and Lee and Cho [10] further extended Banach's contraction principle to fuzzy contractive mappings in Heilpern's sense. In our main result (Theorem 3.1 below) we establish a common fixed-point theorem for a sequence of generalized fuzzy uniformly locally contraction mappings on a complete metric space without the requirement of linearity. This is a generalization of many conventional results of the literature.

Let $\varepsilon \in(0, \infty]$, and $\lambda \in(0,1)$. A metric space $(X, d)$ is said to be $\varepsilon$-chainable if given $\zeta, \xi \in X$, there exists an $\varepsilon$-chain from $\zeta$ to $\xi$ (i.e., a finite set of points $\zeta=\zeta_{0}, \zeta_{1}, \zeta_{2}, \ldots, \zeta_{m}=\xi$ such that $d\left(\zeta_{j-1}, \zeta_{j}\right)<\varepsilon$, for all $\left.j=1,2, \ldots, m\right)$. A mapping $T: X \rightarrow X$ is called an $(\varepsilon, \lambda)$ uniformly locally contractive mapping if $\zeta, \zeta \in X$ and $0<d(\zeta, \zeta)<\varepsilon$, implies $d(T \zeta, T \xi) \leq$ $\lambda d(\zeta, \xi)$. A mapping $T: X \rightarrow W(X)$ is called an $(\varepsilon, \lambda)$ uniformly locally contractive fuzzy mapping if $\zeta, \xi \in X$ and $0<d(\zeta, \xi)<\varepsilon$, imply $d_{\infty}(T(\zeta), T(\xi)) \leq \lambda d(\zeta, \xi)$. We remark that a globally contractive mapping can be regarded as an $(\infty, \lambda)$ uniformly locally contractive mapping and for some special spaces every locally contractive mapping is globally contractive.

Theorem 3.1 Let $\varepsilon \in(0, \infty],(X, d)$ a complete $\varepsilon$-chainable metric space and $\left\{T_{i}\right\}_{i=1}^{\infty}$ a sequence of fuzzy mappings from $X$ into $I^{X}$ such that, for each $\zeta \in X$ and $i \in \mathbb{N}, T_{i}(\zeta)_{1} \in$ $C B(X)$. If

$$
\zeta, \xi \in X, \quad 0<d(\zeta, \xi)<\varepsilon \quad \text { implies } \quad D_{1}\left(T_{i}(\zeta), T_{j}(\xi)\right) \leq \psi(d(\zeta, \xi)) d(\zeta, \xi)
$$

for all $i, j \in \mathbb{N}$, where $\psi:[0, \varepsilon) \rightarrow[0,1)$ is a MT-function, then the sequence $\left\{T_{i}\right\}_{i=1}^{\infty}$ has a common fixed point, i.e., there is $\xi^{*} \in X$ such that $\xi^{*} \in T_{i}\left(\xi^{*}\right)_{1}$, for all $i \in \mathbb{N}$.

Proof Let $\xi_{0}$ be an arbitrary, but fixed element of $X$. Find $\xi_{1} \in X$ such that $\xi_{1} \in T_{1}\left(\xi_{0}\right)_{1}$. Let

$$
\xi_{0}=\zeta_{(1,0)}, \quad \zeta_{(1,1)}, \zeta_{(1,2)}, \ldots, \zeta_{(1, m)}=\xi_{1} \in T_{1}\left(\xi_{0}\right)_{1}
$$

be an arbitrary $\varepsilon$-chain from $\xi_{0}$ to $\xi_{1}$. (We suppose, without loss of generality, that $\zeta_{(1, i)} \neq$ $\zeta_{(1, j)}$, for each $i, j \in\{0,1,2, \ldots, m\}$ with $i \neq j$.)

Since $0<d\left(\zeta_{(1,0)}, \zeta_{(1,1)}\right)<\varepsilon$, we deduce that

$$
\begin{aligned}
D_{1}\left(T_{1}\left(\zeta_{(1,0)}\right), T_{2}\left(\zeta_{(1,1)}\right)\right) & \leq \psi\left(d\left(\zeta_{(1,0)}, \zeta_{(1,1)}\right)\right) d\left(\zeta_{(1,0)}, \zeta_{(1,1)}\right) \\
& <\sqrt{\psi\left(d\left(\zeta_{(1,0)}, \zeta_{(1,1)}\right)\right)} d\left(\zeta_{(1,0)}, \zeta_{(1,1)}\right) \\
& <d\left(\zeta_{(1,0)}, \zeta_{(1,1)}\right)<\varepsilon .
\end{aligned}
$$


Rename $\xi_{1}$ as $\zeta_{(2,0)}$. Since $\zeta_{(2,0)} \in T_{1}\left(\zeta_{(1,0)}\right)_{1}$, using Lemma 2.1 we find $\zeta_{(2,1)} \in T_{2}\left(\zeta_{(1,1)}\right)_{1}$ such that

$$
\begin{aligned}
d\left(\zeta_{(2,0)}, \zeta_{(2,1)}\right) & <\sqrt{\psi\left(d\left(\zeta_{(1,0)}, \zeta_{(1,1)}\right)\right)} d\left(\zeta_{(1,0)}, \zeta_{(1,1)}\right) \\
& <d\left(\zeta_{(1,0)}, \zeta_{(1,1)}\right)<\varepsilon
\end{aligned}
$$

Similarly we may choose an element $\zeta_{(2,2)} \in T_{2}\left(\zeta_{(1,2)}\right)_{1}$ such that

$$
\begin{aligned}
d\left(\zeta_{(2,1)}, \zeta_{(2,2)}\right) & <\sqrt{\psi\left(d\left(\zeta_{(1,1)}, \zeta_{(1,2)}\right)\right)} d\left(\zeta_{(1,1)}, \zeta_{(1,2)}\right) \\
& <d\left(\zeta_{(1,1)}, \zeta_{(1,2)}\right)<\varepsilon
\end{aligned}
$$

Thus we obtain a set $\left\{\zeta_{(2,0)}, \zeta_{(2,1)}, \zeta_{(2,2)}, \ldots, \zeta_{(2, m)}\right\}$ of $m+1$ points of $X$ such that $\zeta_{(2,0)} \in$ $T_{1}\left(\zeta_{(1,0)}\right)_{1}$ and $\zeta_{(2, j)} \in T_{2}\left(\zeta_{(1, j)}\right)_{1}$, for $j=1,2, \ldots, m$, with

$$
\begin{aligned}
d\left(\zeta_{(2, j)}, \zeta_{(2, j+1)}\right) & <\sqrt{\psi\left(d\left(\zeta_{(1, j)}, \zeta_{(1, j+1)}\right)\right)} d\left(\zeta_{(1, j)}, \zeta_{(1, j+1)}\right) \\
& <d\left(\zeta_{(1, j)}, \zeta_{(1, j+1)}\right)<\varepsilon
\end{aligned}
$$

for $j=0,1,2, \ldots, m-1$.

Let $\zeta_{(2, m)}=\xi_{2}$. Thus the set of points $\xi_{1}=\zeta_{(2,0)}, \zeta_{(2,1)}, \zeta_{(2,2)}, \ldots, \zeta_{(2, m)}=\xi_{2} \in T_{2}\left(\xi_{1}\right)_{1}$ is an $\varepsilon$-chain from $\xi_{0}$ to $\xi_{1}$. Rename $\xi_{2}$ as $\zeta_{(3,0)}$. Then by the same procedure we obtain an $\varepsilon$-chain

$$
\xi_{2}=\zeta_{(3,0)}, \quad \zeta_{(3,1)}, \zeta_{(3,2)}, \ldots, \zeta_{(3, m)}=\xi_{3} \in T_{3}\left(\xi_{2}\right)_{1}
$$

from $\xi_{2}$ to $\xi_{3}$. Inductively, we obtain

$$
\xi_{n}=\zeta_{(n+1,0)}, \quad \zeta_{(n+1,1)}, \zeta_{(n+1,2)}, \ldots, \zeta_{(n+1, m)}=\xi_{n+1} \in T_{n+1}\left(\xi_{n}\right)_{1}
$$

with

$$
\begin{aligned}
d\left(\zeta_{(n+1, j)}, \zeta_{(n+1, j+1)}\right) & <\sqrt{\psi\left(d\left(\zeta_{(n, j)}, \zeta_{(n, j+1)}\right)\right)} d\left(\zeta_{(n, j)}, \zeta_{(n, j+1)}\right) \\
& <d\left(\zeta_{(n, j)}, \zeta_{(n, j+1)}\right)<\varepsilon
\end{aligned}
$$

for $j=0,1,2, \ldots, m-1$.

Consequently, we construct a sequence $\left\{\xi_{n}\right\}_{n=1}^{\infty}$ of points of $X$ with

$$
\begin{aligned}
& \xi_{1}=\zeta_{(1, m)}=\zeta_{(2,0)} \in T_{1}\left(\xi_{0}\right)_{1}, \\
& \xi_{2}=\zeta_{(2, m)}=\zeta_{(3,0)} \in T_{2}\left(\xi_{1}\right)_{1}, \\
& \xi_{3}=\zeta_{(3, m)}=\zeta_{(4,0)} \in T_{3}\left(\xi_{2}\right)_{1}, \\
& \vdots \\
& \xi_{n+1}=\zeta_{(n+1, m)}=\zeta_{(n+2,0)} \in T_{n+1}\left(\xi_{n}\right)_{1},
\end{aligned}
$$

for all $n \in \mathbb{N}$. 
For each $j \in\{0,1,2, \ldots, m-1\}$, we deduce from $(2)$ that $\left\{d\left(\zeta_{(n, j)}, \zeta_{(n, j+1)}\right)\right\}_{n=1}^{\infty}$ is a decreasing sequence of non-negative real numbers and therefore there exists $l_{j} \geq 0$ such that

$$
\lim _{n \rightarrow \infty} d\left(\zeta_{(n, j)}, \zeta_{(n, j+1)}\right)=l_{j}
$$

By assumption, $\limsup \operatorname{sul}_{t \rightarrow l_{j}^{+}} \psi(t)<1$, so there exists $n_{j} \in \mathbb{N}$ such that $\psi\left(d\left(\zeta_{(n, j)}, \zeta_{(n, j+1)}\right)\right)<$ $s\left(l_{j}\right)$, for all $n \geq n_{j}$ where $\lim \sup _{t \rightarrow l_{j}^{+}} \psi(t)<s\left(l_{j}\right)<1$.

Now put

$$
M_{j}=\max \left\{\max _{i=1, \ldots, n_{j}} \sqrt{\psi\left(d\left(\zeta_{(i, j)}, \zeta_{(i, j+1)}\right)\right)}, \sqrt{s\left(l_{j}\right)}\right\} .
$$

Then, for every $n>n_{j}$, we obtain

$$
\begin{aligned}
d\left(\zeta_{(n, j)}, \zeta_{(n, j+1)}\right) & <\sqrt{\psi\left(d\left(\zeta_{(n-1, j)}, \zeta_{(n-1, j+1)}\right)\right)} d\left(\zeta_{(n-1, j)}, \zeta_{(n-1, j+1)}\right) \\
& <\sqrt{s\left(l_{j}\right)} d\left(\zeta_{(n-1, j)}, \zeta_{(n-1, j+1)}\right) \\
& \leq M_{j} d\left(\zeta_{(n-1, j)}, \zeta_{(n-1, j+1)}\right) \\
& \leq\left(M_{j}\right)^{2} d\left(\zeta_{(n-2, j)}, \zeta_{(n-2, j+1)}\right) \\
& \leq \cdots \\
& \leq\left(M_{j}\right)^{n-1} d\left(\zeta_{(1, j)}, \zeta_{(1, j+1)}\right) .
\end{aligned}
$$

Putting $N=\max \left\{n_{j}: j=0,1,2, \ldots, m-1\right\}$, we have

$$
\begin{aligned}
d\left(\xi_{n-1}, \xi_{n}\right) & =d\left(\zeta_{(n, 0)}, \zeta_{(n, m)}\right) \leq \sum_{j=0}^{m-1} d\left(\zeta_{(n, j)}, \zeta_{(n, j+1)}\right) \\
& <\sum_{j=0}^{m-1}\left(M_{j}\right)^{n-1} d\left(\zeta_{(1, j)}, \zeta_{(1, j+1)}\right)
\end{aligned}
$$

for all $n>N+1$. Hence

$$
\begin{aligned}
d\left(\xi_{n}, \xi_{p}\right) & \leq d\left(\xi_{n}, \xi_{n+1}\right)+d\left(\xi_{n+1}, \xi_{n+2}\right)+\cdots+d\left(\xi_{p-1}, \xi_{p}\right) \\
& <\sum_{j=0}^{m-1}\left(M_{j}\right)^{n} d\left(\zeta_{(1, j)}, \zeta_{(1, j+1)}\right)+\cdots+\sum_{j=0}^{m-1}\left(M_{j}\right)^{p-1} d\left(\zeta_{(1, j)}, \zeta_{(1, j+1)}\right),
\end{aligned}
$$

whenever $p>n>N+1$.

Since $M_{j}<1$, for all $j \in\{0,1,2, \ldots, m-1\}$, it follows that $\left\{\xi_{n}\right\}_{n=1}^{\infty}$ is a Cauchy sequence. Since $(X, d)$ is complete, there is $\xi^{*} \in X$ such that $\xi_{n} \rightarrow \xi^{*}$. So for each $\delta \in(0, \varepsilon]$ there is $M_{\delta} \in \mathbb{N}$ such that $n>M_{\delta}$ implies $d\left(\xi_{n}, \xi^{*}\right)<\delta$. This in view of inequality (1) implies $D_{1}\left(T_{n+1}\left(\xi_{n}\right), T_{i}\left(\xi^{*}\right)\right)<\delta$, for all $i \in \mathbb{N}$. Consequently, $H\left(T_{n+1}\left(\xi_{n}\right)_{1}, T_{i}\left(\xi^{*}\right)_{1}\right) \rightarrow 0$. Since $\xi_{n+1} \in$ $T_{n+1}\left(\xi_{n}\right)_{1}$ with $d\left(\xi_{n+1}, \xi^{*}\right) \rightarrow 0$, we deduce from Lemma 2.2 that $\xi^{*} \in T_{i}\left(\xi^{*}\right)_{1}$, for all $i \in \mathbb{N}$. This completes the proof. 
Corollary 3.2 Let $\varepsilon \in(0, \infty],(X, d)$ a complete $\varepsilon$-chainable metric space and $\left\{T_{i}\right\}_{i=1}^{\infty} a$ sequence of fuzzy mappings from $X$ into $I^{X}$ such that, for each $\zeta \in X$ and $i \in \mathbb{N}, T_{i}(\zeta)_{1} \in$ $C B(X)$. If

$$
\zeta, \xi \in X, \quad 0<d(\zeta, \xi)<\varepsilon \quad \text { implies } \quad D_{1}\left(T_{i}(\zeta), T_{j}(\xi)\right) \leq \lambda d(\zeta, \xi)
$$

for all $i, j \in \mathbb{N}$, where $\lambda \in(0,1)$, then the sequence $\left\{T_{i}\right\}_{i=1}^{\infty}$ has a common fixed point.

Proof Apply Theorem 3.1 when $\psi$ is the $M T$-function defined as $\psi(t)=\lambda$, for all $t \in$ $[0, \varepsilon)$.

Corollary 3.3 Let $\varepsilon \in(0, \infty],(X, d)$ a complete $\varepsilon$-chainable metric linear space and $\left\{T_{i}\right\}_{i=1}^{\infty}$ a sequence of fuzzy mappings from $X$ into $W(X)$ satisfying the following condition:

$$
\zeta, \xi \in X, \quad 0<d(\zeta, \xi)<\varepsilon \quad \text { implies } \quad d_{\infty}\left(T_{i}(\zeta), T_{j}(\xi)\right) \leq \psi(d(\zeta, \xi)) d(\zeta, \xi),
$$

for all $i, j \in \mathbb{N}$, where $\psi:[0, \varepsilon) \rightarrow[0,1)$ is a MT-function. Then the sequence $\left\{T_{i}\right\}_{i=1}^{\infty}$ has a common fixed point.

Proof Since $W(X) \subseteq C B(X)$ and $D_{1}\left(T_{i}(\zeta), T_{j}(\xi)\right) \leq d_{\infty}\left(T_{i}(\zeta), T_{j}(\xi)\right)$, for all $i, j \in \mathbb{N}$, the result follows immediately from Theorem 3.1.

Corollary 3.4 Let $\varepsilon \in(0, \infty],(X, d)$ a complete $\varepsilon$-chainable metric linear space and $\left\{T_{i}\right\}_{i=1}^{\infty}$ a sequence of fuzzy mappings from $X$ into $W(X)$ satisfying the following condition:

$$
\zeta, \xi \in X, \quad 0<d(\zeta, \xi)<\varepsilon \quad \text { implies } \quad d_{\infty}\left(T_{i}(\zeta), T_{j}(\xi)\right) \leq \lambda d(\zeta, \xi)
$$

for all $i, j \in \mathbb{N}$, where $\lambda \in(0,1)$. Then the sequence $\left\{T_{i}\right\}_{i=1}^{\infty}$ has a common fixed point.

Corollary 3.5 [4] Let $\varepsilon \in(0, \infty],(X, d)$ a complete $\varepsilon$-chainable metric linear space and $T_{1}$, $T_{2}$, two fuzzy mappings from $X$ into $W(X)$ satisfying the following condition:

$$
\zeta, \xi \in X, \quad 0<d(\zeta, \xi)<\varepsilon \quad \text { implies } \quad d_{\infty}\left(T_{i}(\zeta), T_{j}(\xi)\right) \leq \psi(d(\zeta, \xi)) d(\zeta, \xi),
$$

for $i, j=1,2$, where $\psi:[0, \varepsilon) \rightarrow[0,1)$ is a MT-function. Then $T_{1}$ and $T_{2}$ have a common fixed point.

Corollary 3.6 $[4,11]$ Let $\varepsilon \in(0, \infty],(X, d)$ a complete $\varepsilon$-chainable metric linear space and $T: X \rightarrow W(X)$ an $(\varepsilon, \lambda)$ uniformly locally contractive fuzzy mapping. Then $T$ has a fixed point.

Corollary 3.7 Let $\varepsilon \in(0, \infty],(X, d)$ a complete $\varepsilon$-chainable metric space and $S$ be a multivalued mapping from $X$ into $C B(X)$ satisfying the following condition:

$$
\zeta, \xi \in X, \quad 0<d(\zeta, \xi)<\varepsilon \quad \text { implies } \quad H(S(\zeta), S(\xi)) \leq \psi(d(\zeta, \xi)) d(\zeta, \xi)
$$

where $\psi:[0, \varepsilon) \rightarrow[0,1)$ is a MT-function. Then $S$ has a fixed point. 
Proof Define a fuzzy mapping $T$ from $X$ into $I^{X}$ as $T(\xi)(t)=1$ if $t \in S(\xi)$ and $T(\xi)(t)=0$, otherwise. Then $T(\xi)_{1}=S(\xi)$, for all $\xi \in X$, so $T(\xi)_{1} \in C B(X)$, for all $\xi \in X$. Since

$$
D_{1}(T(\zeta), T(\xi))=H\left(T(\zeta)_{1}, T(\xi)_{1}\right)=H(S(\zeta), S(\xi)),
$$

for all $\zeta, \xi \in X$, we deduce that condition (1) of Theorem 3.1 is satisfied for $T$. Hence $T$ has a fixed point $\xi^{*}$, i.e., $\xi^{*} \in T\left(\xi^{*}\right)_{1}$. We conclude that $\xi^{*} \in S\left(\xi^{*}\right)$. The proof is complete.

Corollary 3.8 [13] Let $\varepsilon \in(0, \infty],(X, d)$ a complete $\varepsilon$-chainable metric space and $S$ be a multivalued mapping from $X$ into $C B(X)$ satisfying the following condition:

$$
\zeta, \xi \in X, \quad 0<d(\zeta, \xi)<\varepsilon \quad \text { implies } \quad H(S(\zeta), S(\xi)) \leq \lambda d(\zeta, \xi)
$$

where $\lambda \in(0,1)$. Then $S$ has a fixed point.

Corollary 3.9 ([20, 21], see also $[9,13])$ Let $(X, d)$ be a complete metric space, $S$ a multivalued mapping from $X$ into $C B(X)$ and $\psi:[0, \infty) \rightarrow[0,1)$ a MT-function such that

$$
H(S \zeta, S \xi) \leq \psi(d(\zeta, \xi)) d(\zeta, \xi)
$$

for all $\zeta, \xi \in X$. Then $S$ has a fixed point in $X$.

Proof Apply Corollary 3.8 with $\varepsilon=\infty$.

We conclude the paper with two examples to support Theorem 3.1 and Corollary 3.2.

Example 3.10 Let $(X, d)$ be the compact, and thus complete, metric space such that $X=$ $[0,1]$, and $d(x, y)=|x-y|$, for all $x, y \in X$. Let $\lambda$ be a constant such that $\lambda \in[1 / 14,1)$ and let $\left\{T_{k}\right\}_{k=1}^{\infty}$ be the sequence of fuzzy mappings defined from $X$ into $I^{X}$ as follows:

$$
\begin{aligned}
\text { if } x=0, \quad T_{k}(x)(y) & = \begin{cases}1 & \text { if } y=0, \\
1 / 3 k & \text { if } 0<y \leq 1 / 100, \quad k \in \mathbb{N}, \\
0 & \text { if } 1 / 100<y \leq 1,\end{cases} \\
\text { if } x \neq 0, \quad T_{k}(x)(y) & =\left\{\begin{array}{ll}
1 & \text { if } 0 \leq y \leq x / 14, \\
\lambda / 2 k & \text { if } x / 14<y \leq x / 12, \\
\lambda / 3 k & \text { if } x / 12<y<x, \\
0 & \text { if } x \leq y \leq 1,
\end{array} \quad k \in \mathbb{N} .\right.
\end{aligned}
$$

For each $x, y \in X$ with $x \neq y$, and $i, j \in \mathbb{N}$ we have

$$
D_{1}\left(T_{i}(x), T_{j}(y)\right)=H\left(T_{i}(x)_{1}, T_{j}(y)_{1}\right)=H([0, x / 14],[0, y / 14])=\frac{1}{14}|x-y| .
$$

Hence, for $\psi(t)=\lambda$, the conditions of Corollary 3.2, and hence of Theorem 3.1, are satisfied for any $\varepsilon \in(0, \infty]$, whereas $X$ is not linear. Therefore all previous relevant fixed point results Corollaries 3.3-3.6 on metric linear spaces are not applicable. 
Example 3.11 Let $(X, d)$ be the complete metric space such that $X=[0, \infty), d(x, x)=0$, for all $x \in X$, and $d(x, y)=\max \{x, y\}$ whenever $x \neq y$ (in the sequel we shall write $x \vee y$ instead of $\max \{x, y\})$.

Note that a sequence $\left\{x_{n}\right\}_{n=1}^{\infty}$ is a Cauchy sequence in $(X, d)$ if and only if $d\left(x_{n}, 0\right) \rightarrow 0$. Moreover, $x=0$ is the only non-isolated point of $X$ for the topology induced by $d$.

Let $\psi:[0, \infty) \rightarrow[0,1)$ be the $M T$-function defined as

$$
\psi(t)= \begin{cases}1 / 2 & \text { if } 0 \leq t \leq 1 \\ t /(t+1) & \text { if } t>1\end{cases}
$$

and let $\left\{T_{k}\right\}_{k=1}^{\infty}$ be the sequence of fuzzy mappings defined from $X$ into $I^{X}$ as follows:

$$
\begin{aligned}
& \text { if } 0 \leq x \leq 1, \quad T_{k}(x)(y)=\left\{\begin{array}{ll}
1 & \text { if } x / 4 k \leq y \leq x / 2 k, \\
0 & \text { otherwise }
\end{array} \quad k \in \mathbb{N},\right. \\
& \text { if } x>1, \quad T_{k}(x)(y)=\left\{\begin{array}{ll}
1 & \text { if } x / 2 k \leq y<x^{2} / k(1+x), \\
0 & \text { otherwise, }
\end{array} \quad k \in \mathbb{N} .\right.
\end{aligned}
$$

Observe that, for $0 \leq x \leq 1$,

$$
T_{k}(x)_{1}=\left[\frac{x}{4 k}, \frac{x}{2 k}\right]
$$

and, for $x>1$,

$$
T_{k}(x)_{1}=\left[\frac{x}{2 k}, \frac{x^{2}}{k(1+x)}\right) .
$$

Therefore $T_{k}(x)_{1} \in C B(X)$, for all $x \in X$ and $k \in \mathbb{N}$ (recall that each $x \neq 0$ is an isolated point for the induced topology, so every bounded interval belongs to $C B(X)$ ).

We show that condition (1) of Theorem 3.1 is satisfied for $\varepsilon=\infty$ and $\psi$ as defined above. Indeed, let $x, y \in X$ with $x \neq y$ and $j, k \in \mathbb{N}$. Assume without loss of generality that $x>y$.

If $x, y>1$, for each $b \in T_{j}(y)_{1}$, we obtain

$$
d\left(T_{k}(x)_{1}, b\right)=\inf _{a \in T_{k}(x)_{1}}(a \vee b) \leq \frac{x^{2}}{k(1+x)} \vee b \leq \frac{x^{2}}{k(1+x)} \vee \frac{y^{2}}{j(1+y)}
$$

Similarly, for each $a \in T_{k}(x)_{1}$, we obtain

$$
d\left(a, T_{j}(y)_{1}\right) \leq \frac{x^{2}}{k(1+x)} \vee \frac{y^{2}}{j(1+y)} .
$$

Consequently

$$
\begin{aligned}
D_{1}\left(T_{k}(x), T_{j}(y)\right) & =H\left(T_{k}(x)_{1}, T_{j}(y)_{1}\right) \leq \frac{x^{2}}{k(1+x)} \vee \frac{y^{2}}{j(1+y)} \\
& \leq \frac{(x \vee y)^{2}}{1+(x \vee y)}=\frac{d(x, y)}{1+d(x, y)} d(x, y) \\
& =\psi(d(x, y)) d(x, y) .
\end{aligned}
$$


If $x>1$ and $y \leq 1$, we deduce, in a similar way, that

$$
\begin{aligned}
D_{1}\left(T_{k}(x), T_{j}(y)\right) & =H\left(T_{k}(x)_{1}, T_{j}(y)_{1}\right) \leq \frac{x^{2}}{k(1+x)} \vee \frac{y}{2 j} \\
& \leq \frac{x^{2}}{1+x} \vee \frac{y}{2} \leq \frac{x^{2}}{1+x} \vee \frac{x}{2}=\frac{x^{2}}{1+x} \\
& =\frac{(x \vee y)^{2}}{1+(x \vee y)}=\frac{d(x, y)}{1+d(x, y)} d(x, y) \\
& =\psi(d(x, y)) d(x, y) .
\end{aligned}
$$

Finally, if $x, y \leq 1$, we deduce

$$
\begin{aligned}
D_{1}\left(T_{k}(x), T_{j}(y)\right) & =H\left(T_{k}(x)_{1}, T_{j}(y)_{1}\right) \leq \frac{x}{2 k} \vee \frac{y}{2 j} \\
& \leq \frac{x \vee y}{2}=\psi(d(x, y)) d(x, y) .
\end{aligned}
$$

We have shown that all conditions of Theorem 3.1 are satisfied (in fact $x=0$ is the only fixed point of $T$ ).

\section{Competing interests}

The authors declare that they have no competing interests.

\section{Authors' contributions}

The three authors contributed equally in writing this article. They read and approved the final manuscript.

\section{Author details}

${ }^{1}$ Department of Mathematics, COMSATS Institute of Information Technology, Chak Shahzad, 44000, Pakistan. ${ }^{2}$ Instituto Universitario de Matemática Pura y Aplicada, Universitat Politècnica de València, Valencia, 46022, Spain.

\section{Acknowledgements}

The third author thanks the support of the Ministry of Economy and Competitiveness of Spain, Grant MTM2012-37894-C02-01.

\section{Received: 28 October 2013 Accepted: 20 January 2014 Published: 13 Feb 2014}

\section{References}

1. Heilpern, S: Fuzzy mappings and fixed point theorems. J. Math. Anal. Appl. 83, 566-569 (1981)

2. Ali, B, Abbas, M: Suzuki-type fixed point theorem for fuzzy mappings in ordered metric spaces. Fixed Point Theory Appl. 2013, Article ID 9 (2013)

3. Arshad, M, Azam, A: Fixed points solutions of sequence of locally contractive fuzzy mappings via iterative process. In: International Conference of Mathematical Sciences (ICM), 2012, 11-14 March, Al Ain, UAE (2012)

4. Azam, A, Beg, I: Common fixed points of fuzzy maps. Math. Comput. Model. 49, 1331-1336 (2009)

5. Azam, A, Arshad, M, Beg, l: Fixed points of fuzzy contractive and fuzzy locally contractive maps. Chaos Solitons Fractals 42, 2836-2841 (2009)

6. Azam, A, Arshad, M, Vetro, P: On a pair of fuzzy- $\phi$ contractive mappings. Math. Comput. Model. 52, 207-214 (2010)

7. Azam, A, Arshad, M: A note on 'Fixed point theorems for fuzzy mappings' by P. Vijayaraju and M. Marudai. Fuzzy Sets Syst. 161, 1145-1149 (2010)

8. Azam, A, Waseem, M, Rashid, M: Fixed point theorems for fuzzy contractive mappings in quasi-pseudo-metric spaces. Fixed Point Theory Appl. 2013, Article ID 27 (2013)

9. Kamran, T: Common fixed points theorems for fuzzy mappings. Chaos Solitons Fractals 38, 1378-1382 (2008)

10. Lee, BS, Cho, SJ: A fixed point theorem for contractive type fuzzy mappings. Fuzzy Sets Syst. 61, 309-312 (1994)

11. Hu, T: Fixed point theorems for multivalued mappings. Can. Math. Bull. 23, 193-197 (1980)

12. Du, WS: On coincidence point and fixed point theorems for nonlinear multivalued maps. Topol. Appl. 159, 49-56 (2012)

13. Edelstein, M: An extension of Banach's contraction principle. Proc. Am. Math. Soc. 12, 7-12 (1961)

14. Beg, I, Azam, A: Fixed points of multivalued locally contractive mappings. Boll. Unione Mat. Ital., A (7) 7, 227-233 (1990)

15. Holmes, RD: On fixed and periodic points under certain set of mappings. Can. Math. Bull. 12, 813-822 (1969)

16. Hu, T, Rosen, H: Locally contractive and expansive mappings. Proc. Am. Math. Soc. 86, 656-662 (1982) 
17. Ko, HM, Tasi, YH: Fixed point theorems for localized property. Tamkang J. Math. 8, 81-85 (1977)

18. Kuhfitting, PK: Fixed point of locally contractive and nonexpansive set valued mappings. Pac. J. Math. 65, 399-403 (1976)

19. Nadler, SB: Multivalued contraction mappings. Pac. J. Math. 30, 475-488 (1969)

20. Mizoguchi, N, Takahashi, W: Fixed point theorems for multi-valued mappings on complete metric spaces. J. Math. Anal. Appl. 188, 141-177 (1989)

21. Suzuki, T: Mizoguchi-Takahashi's fixed point theorem is a real generalization of Nadler's. J. Math. Anal. Appl. 340, $752-755$ (2008)

10.1186/1029-242X-2014-74

Cite this article as: Ahmad et al.: On locally contractive fuzzy set-valued mappings. Journal of Inequalities and Applications 2014, 2014:74

Submit your manuscript to a SpringerOpen ${ }^{\odot}$ journal and benefit from:

- Convenient online submission

Rigorous peer review

- Immediate publication on acceptance

- Open access: articles freely available online

- High visibility within the field

- Retaining the copyright to your article 